\title{
Single breath-hold slice-following CSPAMM myocardial tagging
}

\author{
M. Stuber ${ }^{\mathrm{a}, \mathrm{b}, *}$, M.A. Spiegel ${ }^{\mathrm{a}}$, S.E. Fischer ${ }^{\mathrm{a}}$, M.B. Scheidegger ${ }^{\mathrm{a}}$, P.G. Danias ${ }^{\mathrm{b}}$, \\ E.M. Pedersen ${ }^{c}$, P. Boesiger ${ }^{a}$ \\ "Institute of Biomedical Engineering and Medical Informatics, University and ETH Zurich, Zurich. Switzerland \\ ${ }^{b}$ Department of Medicine, Cardiotascular Division, Beth Israel Deaconess Medical Center and Hartard Medical School, 330 Brookline Ate., \\ Boston, MA 02215, USA \\ "Department of Cardiothoracic and Vascular Surgery, Skeiby Sygehus, Aarhus Laicersity Hospital, Aarhus, Denmark
}

Received 17 May 1999: accepted 30 July 1999

\begin{abstract}
Myocardial tagging has shown to be a useful magnetic resonance modality for the assessment and quantification of local myocardial function. Many myocardial tagging techniques suffer from a rapid fading of the tags, restricting their application mainly to systolic phases of the cardiac cycle. However, left ventricular diastolic dysfunction has been increasingly appreciated as a major cause of heart failure. Subtraction based slice-following CSPAMM myocardial tagging has shown to overcome limitations such as fading of the tags. Remaining impediments to this technique, however, are extensive scanning times ( $\sim 10$ min), the requirement of repeated breath-holds using a coached breathing pattern, and the enhanced sensitivity to artifacts related to poor patient compliance or inconsistent depths of end-expiratory breath-holds. We therefore propose a combination of slice-following CSPAMM myocardial tagging with a segmented EPI imaging sequence. Together with an optimized RF excitation scheme, this enables to acquire as many as 20 systolic and diastolic grid-tagged images per cardiac cycle with a high tagging contrast during a short period of sustained respiration. (C) 1999 Elsevier Science B.V. All rights reserved.
\end{abstract}

Keywords: CSPAMM: Myocardial tagging: Myocardial motion analysis: Diastolic function; Torsion; Slice following

\section{Introduction}

Myocardial tagging has shown to be a useful magnetic resonance (MR) modality for the assessment and quantification of local myocardial function [1-8] in the healthy and in the diseased state. This technology is also gaining greater use in the assessment of time dependent changes due to its non-invasive acquisition and ease for serial assessments at rest and during stress [4].

Many myocardial tagging techniques suffer from the rapid fading of the tags which may not be reliably detected after end-systole [9]. However, left ventricular diastolic dysfunction has been increasingly appreciated

\footnotetext{
* Corresponding author. Tel.: + 1-617-6678037; fax: + 1-6179755480.

E-mail address: mstuber@caregroup.harvard.edu (M. Stuber)
}

as a major cause of heart failure $[10,11]$. Subtraction based CSPAMM [12] is a technique that prolongs the persistence of the tags into diastole and has been extended with a slice-following concept to avoid misregistration in short axis views [13] due to base-to-apex shortening [14]. These two advantages of CSPAMM have recently been shown to be most valuable for clinical patient studies in which systolic and diastolic left ventricular torsion was investigated [15]. Major limitations to this technique are extensive scanning times, the requirement of repeated breath holds using a coached breathing pattern and the enhanced sensitivity to artifacts related to poor patient compliance or inconsistent depths of end-expiratory breath-holds. While successful breath-hold tagging techniques have already been described [9], single breath-hold techniques in conjunction with slice-following CSPAMM MR myocardial tagging have not previously been reported. 


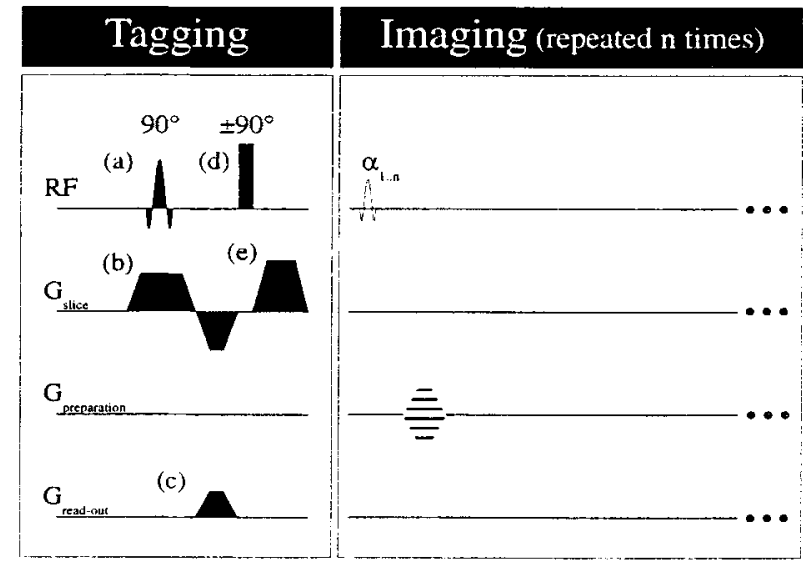

Fig. 1. CSPAMM myocardial tagging procedure in combination with a multi heart-phase segmented ccho planar imaging (EPI) sequence. After slice selection (RF pulse (a) and gradient (b)), the transverse magnetization is spoiled with the tagging gradient (c). The RF block-pulse (d) rotates the modulated transverse magnetization into positive or negative z-direction. Gradient (e) finally spoils the remaining transverse magnetization. For the imaging part of the sequence. the EPI readout is performed for $\mathrm{n}$ heart-phases with corresponding RF excitation angles $x_{1 \ldots, n}$ for imaging.

\section{Methods}

\subsection{Background}

For cardiac applications, tissue tagging (or the spatially dependent modulation of the magnetization) is typically applied after the detection of the R-wave of the ECG. Subsequently, different phases of the cardiac cycle are imaged using a multi heart-phase imaging sequence.

The following considerations apply for multi heartphase imaging sequences in which one RF excitation for imaging is applied per heart-phase and $\mathrm{k}$-space segment. For a CSPAMM experiment in which $n$ heart- phase images are acquired per cardiac cycle (Fig. 1), the signal intensity $I_{T k}$ for the $k$ th $(k=1 \ldots n)$ heart-phase image has been described [12] as

$$
I_{T k} \propto M_{\mathrm{ss}} T A G(x, y) \exp (-\Delta t / T 1) \prod_{j=1}^{k-1} \cos \left(x_{j}\right) \sin \left(x_{k}\right)
$$

where $M_{\mathrm{ss}}$ is the steady state magnetization; $T A G(x, y)$ is the spatially dependent $(x, y)$ modulation of the magnetization; $\Delta t$ is the interval between the heartphase images (i.e. temporal resolution); $\alpha_{1} \ldots \alpha_{n}$ are the RF excitation angles (Figs. 1 and 2). The signal intensity $I_{T K}$ in Eq. (1) is (a) scaled by the tissue specific time constant $T 1$ and (b) scaled by the RF excitation angles which were applied for imaging. To obtain a constant signal intensity for each acquired image $\left(I_{T k}=I_{T k-1}\right)$, these two effects need to be compensated by the application of the ramped flip angle series [14]:

$x_{k-1}=a \tan \left(\sin \left(\alpha_{k}\right) \exp (-\Delta t / T 1)\right.$

Using a repetitive, coached breathing pattern in which signal sampling is performed during a short, sustained breath-hold period every fourth heartbeat, an $x_{n}=90^{\circ}$ was proposed [14] and has been utilized successfully [15] as a starting angle for the recursion in Eq. (2).

However, for single breath-hold techniques in which signal sampling during each cardiac cycle would be preferable, the application of $\alpha_{n}=90^{\circ}$ is not practical, since the steady state magnetization and consequently the signal-to-noise ratio (SNR) and contrast-to-noise ratio (CNR) would be compromised due to a shortened relaxation time $t_{\text {relax }}$ (Fig. 2). Thus, the $R F$ excitation angle series $x_{1} \ldots x_{n}$ needs to be adapted for single breath-hold CSPAMM applications.

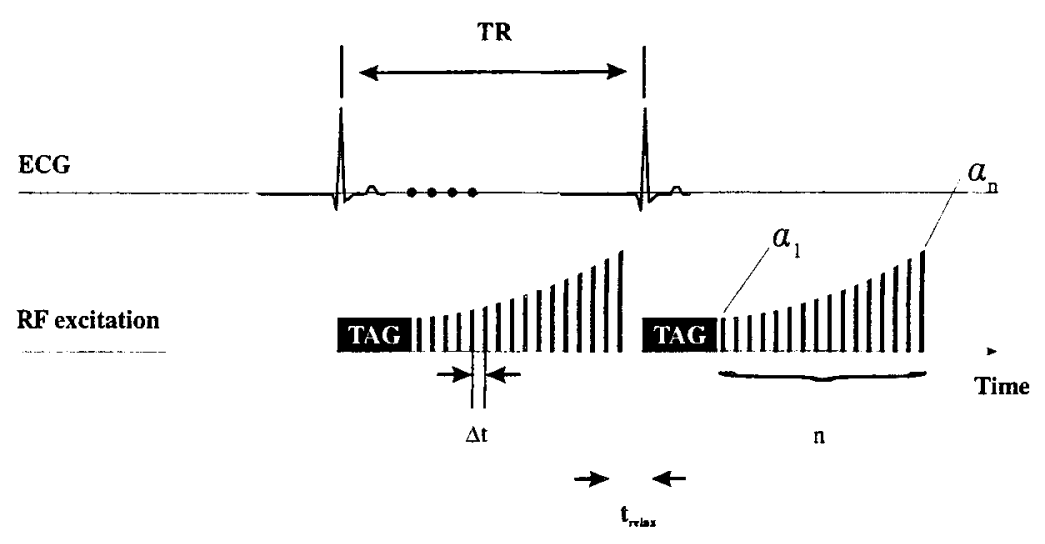

Fig. 2. Temporal relationship between the R-wave of the ECG, the tagging part of the sequence and the imaging part of the sequence. The tagging procedure is performed typically after the detection of the R-wave of the ECG. The time-interval in-between the tagging procedures is $T R$. The time delay between the $n$ heart-phase images is $\Delta t$ and the delay between the last RF excitation for imaging and the subsequent tagging procedure is $t_{\text {relax }}$. The variable RF excitation angles for imaging are denoted as $x_{1} \ldots x_{n}$. 


\subsection{Numerical simulation}

To maximize the relative signal amplitude, $I_{T h}$, of Eq. (1) as a function of the RF excitation angles, a numerical simulation using Eqs. (1) and (2) was implemented under PV-Wave ${ }^{(\mathcal{Q})}$ 6.0, (Visual Numerics, Houston, TX). The simulation included a multi heart-phase tagging experiment in which acquisition of 20 images per cardiac cycle were simulated. The ranges of the parameters utilized in the simulation were $0-90^{\circ}$ for the initial RF excitation $\alpha_{n}, 0-100 \mathrm{~ms}$ for the time resolution $\Delta t$ and $500-4000 \mathrm{~ms}$ for the repetition time $T R$. A myocardial muscle $T 1$ of $850 \mathrm{~ms}$ was assumed.

\subsection{Implementation}

Single breath-hold slice-following CSPAMM myocardial tagging was implemented on a Philips Gyroscan ACS-NT 1.5T system (Philips Medical Systems, Best, NL) equipped with a CPR6 extended cardiac software package, a five element cardiac synergy coil and a PowerTrak6000 gradient system $(23 \mathrm{mT} / \mathrm{m} ; 105$ $\mathrm{mT} / \mathrm{m}$ per $\mathrm{ms})$. A multi heart-phase segmented echoplanar imaging sequence with variable RF excitation flip angles was combined with the tagging part of the sequence (Figs. 1 and 2). The excitation angle $\alpha_{n}$ can be adjusted interactively by the operator. The dependent RF excitation angles $\alpha_{n-1} \ldots \alpha_{1}$ are then calculated automatically by the scanner software according to Eq. (2). This implementation takes advantage of a reduced $k$ space acquisition scheme [9] in which horizontally and vertically line-tagged images are both acquired during a single breath-hold while the line orientation is automatically changed during the breath-hold period.

The thickness of the imaged volume $(d s)$ and the offcenter of the tagged slice with respect to the imaged volume $\left(z_{0}\right)$ can be adjusted to account for variable amounts of systolic base-to-apex shortening at different anatomical levels of the heart (Fig. 3).

\subsection{Examination protocol for in vivo applications}

Six male volunteers (age $28 \pm 6$ years) were investigated in the supine position using ECG triggering. In each individual, grid-tagged cine images were acquired on an apical, a mid and a basal myocardial level. In addition, line tagged images were acquired in a fourchamber view. For each slice, the volunteers were instructed to hold their breath at end-expiration during 12 heartbeats. Twenty images were acquired with a temporal resolution $(\Delta t)$ of $35 \mathrm{~ms}$ and a tag line distance of $8 \mathrm{~mm}$. In one volunteer, a supplementary scan with an $\alpha_{n}$ of $90^{\circ}$ was applied for comparison.

The field of view (FOV) was $300 \mathrm{~mm}$ with a sampled matrix of 128 data points in read-out direction. The thickness of the tagged slice (dz, Fig. 3) was $8 \mathrm{~mm}$.

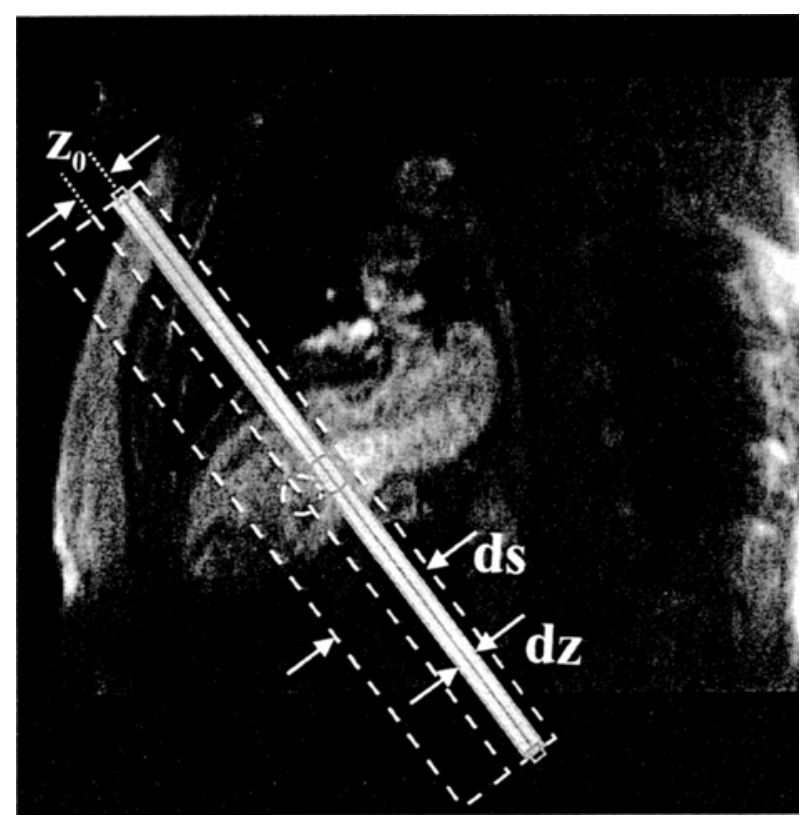

Fig. 3. Planning of a double oblique short axis tagging experiment at a basal level of the heart. A single oblique end-diastolic long axis view is overlaid by the graphic elements which are interactively used for localization. The gray, solid rectangle refers to the tagged slice of the thickness $d z$. The hatched rectangle shows the volume which is imaged. This imaged volume has to encompass the extent of the motion (base to apex shortening) of the initially labeled thin slice during the cardiac cycle. The thickness of this imaged volume is $d s$ and the offcenter of the center of the tagged thin slice with respect to the center of the imaged volume is $z_{0}$.

With a reduced $k$-space [9] acquisition scheme $(25 \%$ of $k_{y}$-profiles), an in-plane resolution of $2.3 \times 2.3 \mathrm{~mm}$ is obtained after multiplication of the horizontally and vertically line tagged heart-phase images. After each RF excitation applied for imaging, nine echo-planar read-outs with a $T E$ of $5.4 \mathrm{~ms}$ were performed (Fig. 1). The thickness ( $d s$ ) of the imaged volume (Fig. 3) was 30 $\mathrm{mm}$ at the base, $25 \mathrm{~mm}$ at the mid-ventricle and $20 \mathrm{~mm}$ at the apex of the heart. For the four-chamber acquisition, the thickness of the imaged volume was $15 \mathrm{~mm}$. The offset $\left(z_{0}\right)$ of the tagged slice in relation to the imaged volume (Fig. 3) was $12 \mathrm{~mm}$ at the base, $6 \mathrm{~mm}$ at the mid-ventricle and $0 \mathrm{~mm}$ at the apex. For the four chamber acquisition, no offset between the tagged and the imaged volume offcenters was used.

\subsection{Image analysis}

In all volunteers, the tagging contrast (CNR) was evaluated in a user specified region-of-interest (ROI) in the intraventricular septum. The difference between maximum and minimum signal within the septum was related to the noise level as determined from an ROI anterior to the sternum ( = standard deviation of the signal in this region). This analysis was performed for 
all four anatomical levels and for each heart phase image.

\section{Results}

\subsection{Numerical simulation}

Fig. 4, displays the results from the numerical simulation of the Eqs. (1) and (2) using a heart-phase interval
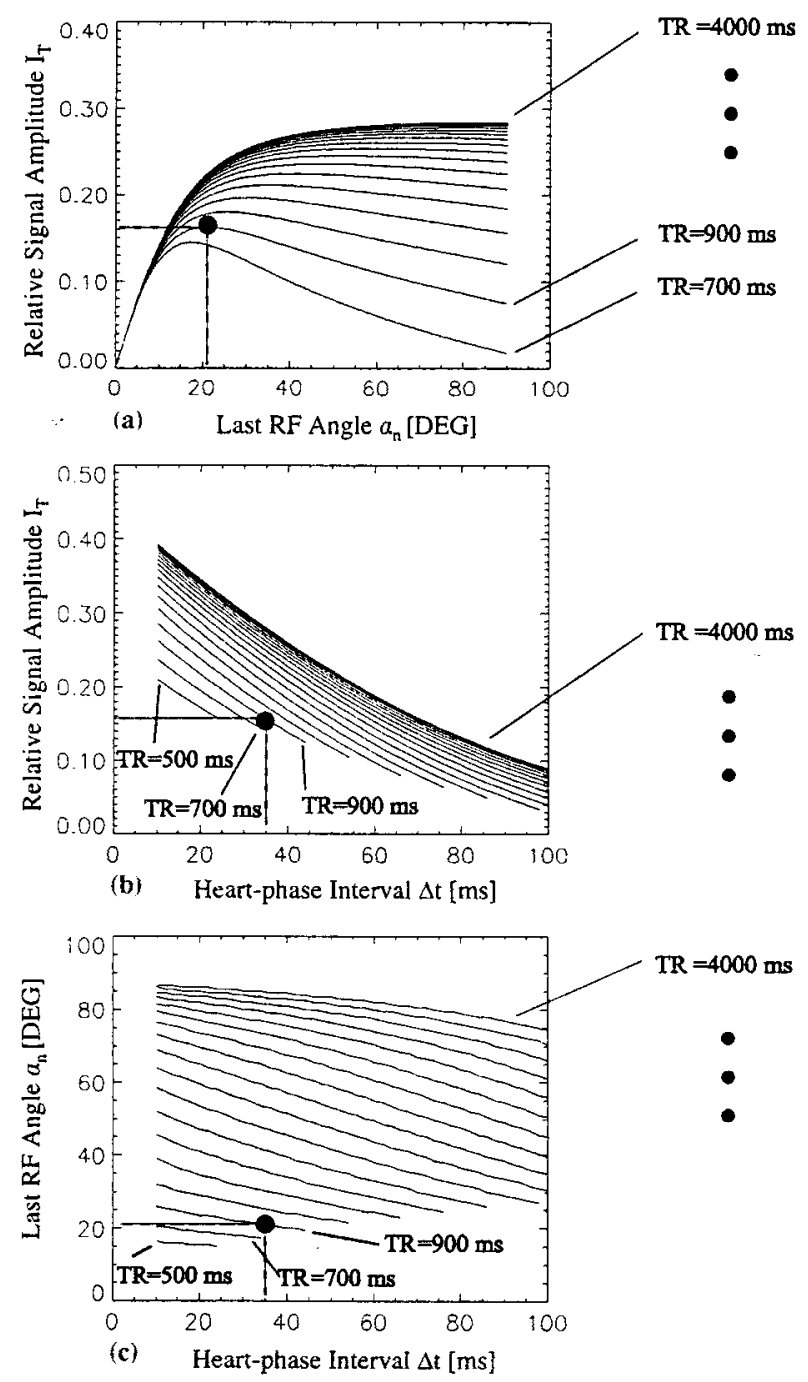

Fig. 4. Signal optimization for $n=20$ heart-phase images. (A) Relative signal amplitude derived from the tagged component of the magnetization. The relative signal amplitude is plotted as function of the last RF excitation angle for imaging $\left(\alpha_{n}\right)$. The data are plotted for a fixed heart-phase interval $\Delta t$ of $35 \mathrm{~ms}$ taking multiple repetition times $T R$ into account. The black dot indicates maximum signal intensity for a repetition time of $900 \mathrm{~ms}$. Maximum signal intensity is ensured if $\alpha_{n}=21^{\circ}$. (B) Maximum relative signal amplitude which can be obtained by an optimized $x_{n}$. It is plotted as a function of $\Delta t$ and $T R$. (C) Optimized $\alpha \mathrm{n}$ as a function of $\Delta t$ and $T R$ (optimized for maximum relative signal intensity from the tagged component of the magnetization). of $35 \mathrm{~ms}$ and 20 heart-phase images per cardiac cycle. In Fig. 4A, the relative signal amplitude of the tagged images is shown as a function of the last RF excitation angle $\alpha_{n}$ and the repetition time $T R$.

For coached breathing patterns in which image data are collected every fourth heartbeat ( $T R \approx 4000 \mathrm{~ms}$ ), an $\alpha_{n}=90^{\circ}$ applied to the recursion shown in Eq. (2) yields a maximized relative signal amplitude $\left(I_{T k} \approx 0.29\right)$. This is consistent with previous findings [14]. If $T R$ is reduced to time periods as typically required for single breath-hold experiments ( $T R<1000 \mathrm{~ms}$ ), an $\alpha_{n}$ of $90^{\circ}$ reduces significantly the relative signal amplitude. For such shortened $T R$ values, maximum signal is obtained if an $\alpha_{n}$ of $21^{\circ}$ is applied (Fig. 4A, black dot).

In Fig. 4B, the maximum relative signal amplitude (which may be obtained by the use of optimized RF excitation angles) is displayed as a function of the heart phase interval $(\Delta t)$ and the repetition time $(T R)$. Enhanced relative signal amplitude is predicted for prolonged $T R$ and shorter heart-phase intervals. Increasing $\Delta t$ and/or decreasing $T R$ will result in signal reduction. Consistent with the findings of Fig. $4 \mathrm{~A}$, the relative signal amplitude amounts to 0.16 if an optimized $\alpha_{n}$ of $21^{\circ}$ is utilized in combination with a $T R$ of $900 \mathrm{~ms}$ and a $\Delta t$ of $35 \mathrm{~ms}$.

While the Fig. 4A and B display relative signal amplitudes as a function of various imaging parameters, Fig. 4C shows the optimized RF excitation angles $\alpha_{n}$ which need to be applied for given $\Delta t$ and $T R$ values. Most obviously, lower RF excitation angles are preferred for acquisition techniques in which data are acquired during successive cardiac cycles $(T R<1000$ ms). For a prolonged $T R$, the $\alpha_{n}$ value may be increased.

\subsection{Images}

In Fig. 5, all 20 heart-phase images acquired at an apical level of the heart in a volunteer (heart rate $=70$ ) are displayed. The time series starts in early systole (35 ms after the R-wave of the ECG) and ends at $700 \mathrm{~ms}$ in diastole. The grid structure remains visible with a high tagging contrast up to the last acquired heart-phase image. No fading of the tags or degradation of image quality is observed. In Fig. 6, three heart-phase images from a four chamber view are displayed. Note the vigorous base to apex shortening of the basal right ventricle during systole (Fig. 5B, arrows, dotted line) with little movement at the apex (dashed line). Fig. 7 visualizes potential adverse effects on image quality which may occur if the RF excitation angles are utilized without optimization. The basal images displayed in Fig. 7 (upper panel) were acquired with an $x_{n}$ of $90^{\circ}$. For comparison, the basal images in the lower panel were acquired with the optimized RF excitation scheme. The degradation in image quality is apparent 

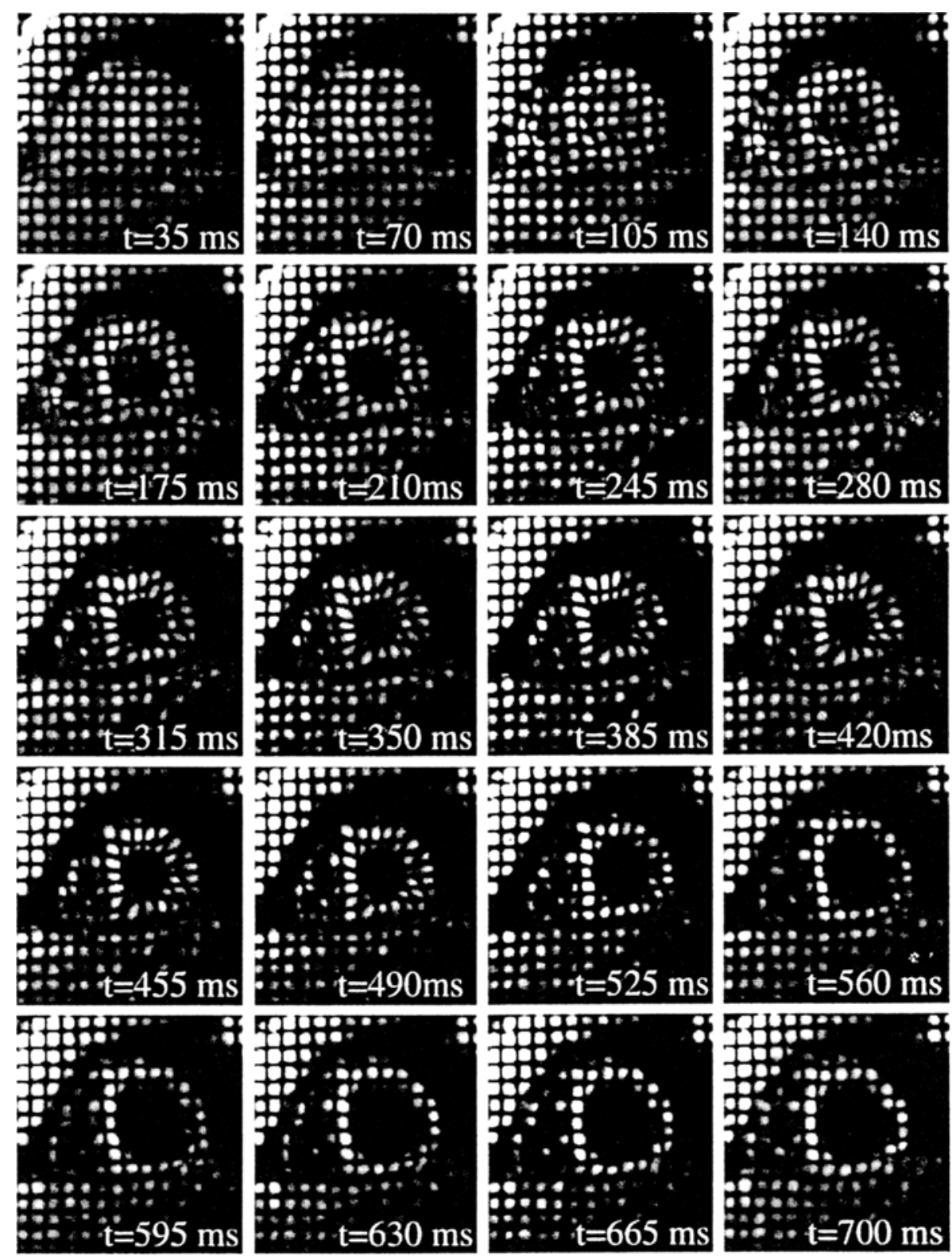

Fig. 5. Twenty apical phases of the cardiac cycle imaged in a double oblique short axis plane of the myocardium (healthy volunteer). The images are acquired using single-breath-hold slice-following CSPAMM myocardial tagging. The acquisition duration was 12 cardiac cycles. The heart-phase interval (time resolution) $\Delta t$ was $35 \mathrm{~ms}$.and the heart rate 70 beats $/ \mathrm{min}$

in this case if non-optimized RF excitation angles are utilized (upper panel).

\subsection{Tagging contrast}

The results obtained from the evaluation of the tagging CNR at the apex, at the mid-ventricle at the base and in the four-chamber view are displayed in Fig. 8A. The tagging CNR for all four anatomical levels is plotted versus time. Consistently, a high tagging CNR is observed for all anatomical levels and for all the acquired heart phase images. In Fig. 8B, the tagging
CNR averaged over all measured anatomical levels including the standard deviations are given as a function of the measured heart-phase image. The average tagging CNR over the cardiac cycle was $19.1 \pm 2.6$. A tendency of an increasing tagging CNR as a function of the measured heart-phase image can be observed.

\section{Discussion}

By the combination of slice-following CSPAMM myocardial tagging with the proposed RF excitation 


\section{End-diastole}

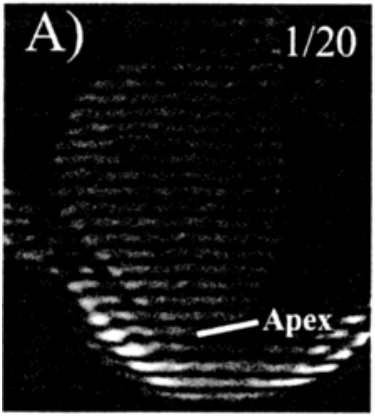

End-systole

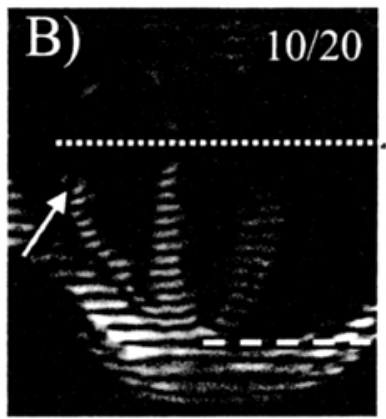

Late diastole

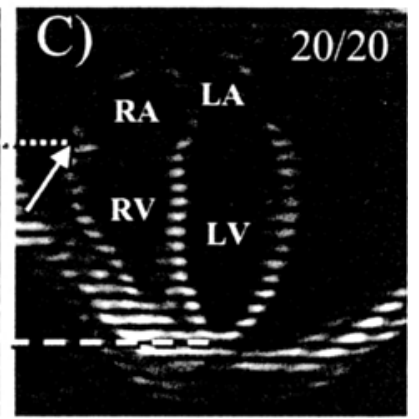

Fig. 6. Four chamber view at three different points in the cardiac cycle. Three out of 20 images are displayed. Image (A) refers to end-diastole, image (B) to end systole and image $(C)$ to diastole. Note the substantial base to apex shortening of the basal right ventricle during systole (dotted line and arrows). The apex remains almost at the same position (dashed line). RA, right atrium: RV, right ventricle; LA. left atrium: LV, left ventricle.

scheme and a segmented EPI imaging sequence, as many as 20 grid-tagged heart-phase images per cardiac cycle can be acquired reliably during one short period of sustained respiration. With respect to earlier CSPAMM studies, this yields a substantially increased coverage of the cardiac cycle $[14,15]$ while image acquisition duration is reduced typically from several minutes to $12 \mathrm{~s}$.

The tagging CNR is consistently high for both systolic and diastolic phases of the cardiac cycle. Since this applies for all the measured anatomical levels of the heart, the most sensitive [15] and fastest occurring motion components such as systolic torsion and diastolic untwist can be assessed within one imaging procedure.

Due to the slice following capabilities of the sequence, adverse effects of through plane motion as induced by systolic base-to-apex shortening (Fig. 6) may be avoided since always the same tissue elements are displayed in the multiple heart-phase images. This enables true myocardial motion tracking [14].

Breath-hold slice-following CSPAMM myocardial tagging is based on a subtraction technique. Thus, the method has a potentially enhanced sensitivity on image artifacts related to inconsistent depths of subsequent breath-holds [16]. Since the grid-tagged cine images of one anatomical level are now referring to one single breath-hold, such sensitivity may be minimized. Still, diaphragmatic drift during prolonged periods of sustained respiration has been reported [16] and could be a limiting factor. Hereby, the combination of CSPAMM myocardial tagging with prospective real-time navigator tracking could be a valuable enhancement [17] of the present methodology. However, this remains to be further investigated.

The present data suggest that the tagging contrast is increasing as a function of the acquired heart-phase image. This may partly be explained by unspoiled transverse magnetization (which was not included in the numerical simulation) or a slight underestimation of myocardial muscle $T 1$ ( $850 \mathrm{~ms}$ ) which may additionally contribute to the signal of later heart-phase images [18].

A further reduction in scanning time or breath-hold duration may be obtained by utilizing faster gradient systems, by a reduction of the image resolution or by the application of hybrid imaging techniques [19]. Realtime applications, however, are not likely to be feasible utilizing CSPAMM myocardial tagging since the technique inherently requires two acquisitions for subtraction.

\section{End-systole Late diastole}
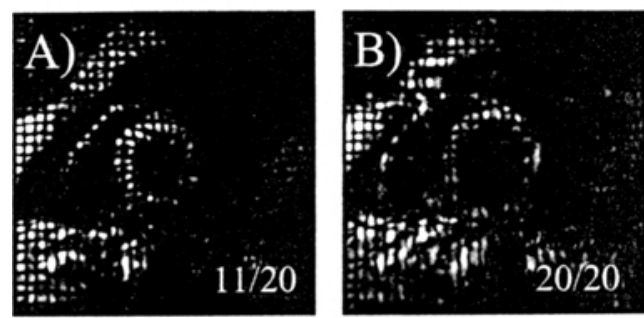

$a_{\mathrm{n}}=90^{\circ}$
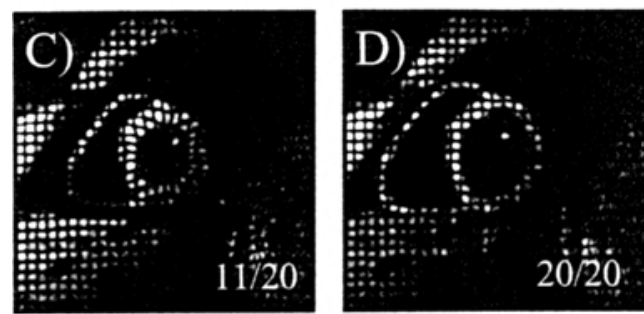

$a_{\mathrm{n}}=21^{\circ}$

Fig. 7. Effect of RF-excitation optimization for slice-following CSPAMM myocardial tagging. Two heart-phase images (end systole and end-diastole) out of 20 are displayed for an $\alpha_{n}$ of $90^{\circ}$ (upper panel) and an optimized $x_{n}$ of $21^{\circ}$ (lower panel). 

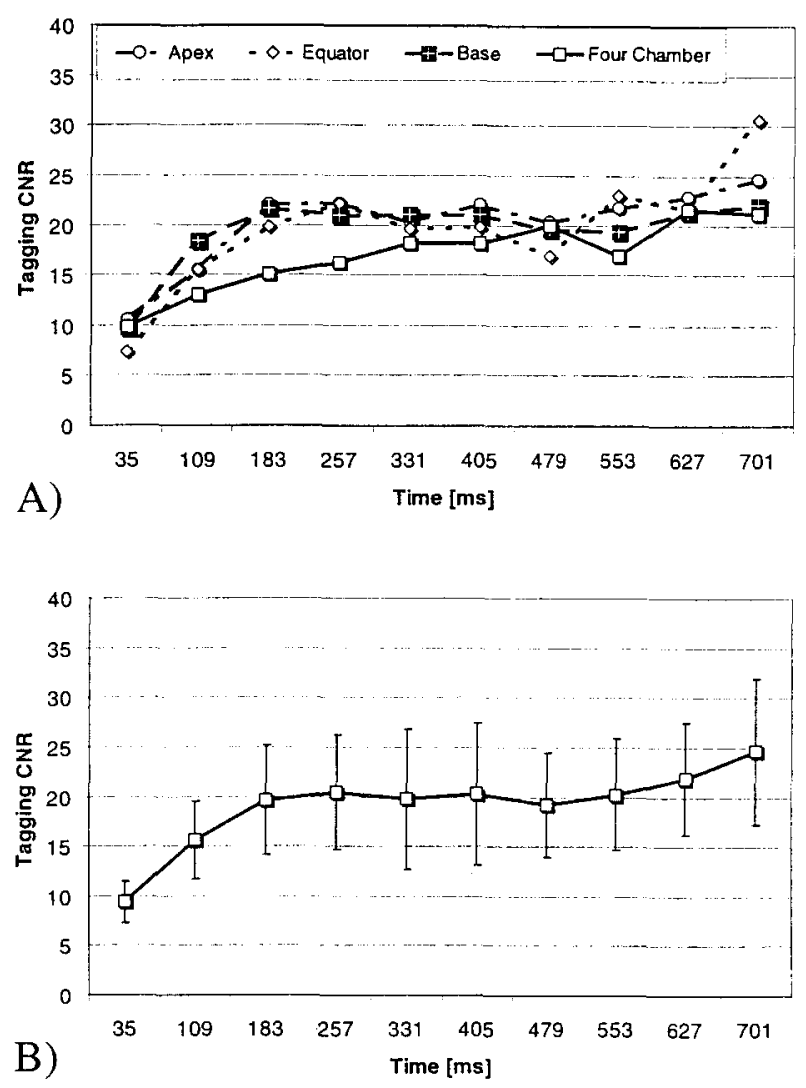

Fig. 8. Tagging CNR assessed in six volunteers as a function of time in the cardiac cycle. (A) Tagging CNR for all the measured anatomical levels. (B) The average tagging contrast of apex. the mid-myocardium, the base and the four chamber view are displayed including \pm one standard deviation.

\section{Conclusions}

The present implementation of slice-following CSPAMM myocardial tagging allows for grid-tagged image acquisition on multiple anatomical levels of the heart during a short period of sustained respiration. Hereby, systolic and diastolic phases of the cardiac cycle can be assessed reliably with a high temporal resolution. This is an important condition for the analysis of systolic and diastolic function in the normal and in the diseased state [15].

By the reduction of the scanning time from several minutes to $12 \mathrm{~s}$ per anatomical level, the advantages of slice-following CSPAMM myocardial tagging are preserved and the susceptibility of the method to artifacts as associated with inconsistent depths of subsequent breath-holds is minimized. This makes the measurement of systolic and diastolic myocardial wall motion a fast, simple, and reproducible exam which may lead to an enhanced clinical applicability of the method.

\section{References}

[1] Zerhouni EA, Parish DM, Rogers WJ, Yang A, Shapiro EP. Human heart: tagging with MR imaging - a method for noninvasive assessment of myocardial motion. Radiology 1988;169:59-63.

[2] Axel L. Dougherty L. MR imaging of motion with spatial modulation of magnetization. Radiology 1989;171:841-5.

[3] Buchalter MB. Weiss JL, Rogers WJ, et al. Noninvasive quantification of left ventricular rotational deformation in normal humans using magnetic resonance imaging myocardial tagging. Circulation 1990:81:1236-44.

[4] McVeigh ER. MRI of myocardial function: motion tracking techniques. Magn Reson Imaging 1996;14:137-50

[5] Clark NR, Reichek N. Bergey P, et al. Circumferential myocardial shortening in the normal human left ventricle. Assessment by magnetic resonance imaging using spatial modulation of magnetization. Circulation 1991:84:67-74.

[6] Wyman BT, Hunter WC, Prinzen FW, McVeigh ER, Mapping propagation of mechanical activation in the paced heart with MRI tagging. Am J Physiol 1999:276:H881-91.

[7] Rogers WJ Jr. Kramer CM, Geskin G, et al. Early contrast-enhanced MRI predicts late functional recovery after reperfused myocardial infarction [see comments]. Circulation 1999;99:74450.

[8] Bogaert J, Maes A. Van de Werf F, et al. Functional recovery of subepicardial myocardial tissue in transmural myocardial infarction after successful reperfusion: an important contribution to the improvement of regional and global left ventricular function. Circulation 1999:99:36-43.

[9] McVeigh ER. Atalar E. Cardiac tagging with breath-hold cine MRI. Magn Reson Med 1992;28:318-27.

[10] Hess OM, Lavelle JF. Sasayama S, Kemper WS, Ross J. Diastolic myocardial wall stiffness of the left ventricle in chronic pressure overload. Eur Heart J 1982:3:315-24.

[11] Hess OM, Villari B, Krayenbuehl HP. Diastolic dysfunction in aortic stenosis. Circulation 1993;87:IV73-6.

[12] Fischer SE, McKinnon GC, Maier SE, Boesiger P. Improved myocardial tagging contrast. Magn Reson Med 1993;30:191200.

[13] Rogers WJ Jr, Shapiro EP, Weiss JL, et al. Quantification of and correction for left ventricular systolic long- axis shortening by magnetic resonance tissue tagging and slice isolation. Circulation 1991:84:721-31

[14] Fischer SE. McKinnon GC. Scheidegger MB. Prins W, Meier D, Boesiger P. True myocardial motion tracking. Magn Reson Med 1994;31:401-13.

[15] Stuber M, Scheidegger MB, Fischer SE, et al. Alterations in the local myocardial motion pattern in patients suffering from pressure overload due to aortic stenosis. Circulation 1999:100:361-8.

[16] Danias PG, Stuber M, Botnar RM, Kissinger KV, Chuang ML. Manning WJ. Breath-hold duration: use of magnetic resonance navigators to assess the impact of supplemental oxygen and hyperventilation. Am J Roentgenol 1998;171:395-7.

[17] Chuang ML, Chen MH, Khasgiwala VC, McConnell MV, Edelman RR, Manning WJ. Adaptive correction of imaging plane position in segmented $k$-space cine cardiac MRI. J Magn Reson Imaging 1997;7:811-4.

[18] Fischer SE. In: Zurich E. editor. Assessment of human heart wall motion by magnetic resonance imaging. Diss.; vol. 10926. Zurich: ETH Zurich, 1994.

[19] Spiegel M, Scheidegger MB, Stuber M, Boesiger P. Ultrafast and real time myocardial motion assessment by tagging. Proc Int Soc Magn Reson Med 1998:1:279. 